\title{
Contemporary Analysis of Erectile, Voiding, and Oncologic Outcomes Following Primary Targeted Cryoablation of the Prostate for Clinically Localized Prostate Cancer
}

\author{
Christopher J. DiBlasio, Ithaar H. Derweesh, John B. Malcolm, Michael M. Maddox, Michael \\ A. Aleman, Robert W. Wake
}

Department of Urology, University of Tennessee Health Science Center, Memphis, Tennessee, USA

\begin{abstract}
Purpose: To evaluate erectile function (EF) and voiding function following primary targeted cryoablation of the prostate (TCAP) for clinically localized prostate cancer $(\mathrm{CaP})$ in a contemporary cohort.

Materials and Methods: We retrospectively reviewed all patients treated between 2/2000-5/2006 with primary TCAP. Variables included age, Gleason sum, pre-TCAP prostate specific antigen (PSA), prostate volume, clinical stage, preTCAP hormonal ablation, pre-TCAP EF and American Urologic Association Symptom Score (AUASS). EF was recorded as follows: $1=$ potent; $2=$ sufficient for intercourse; $3=$ partial $/$ insufficient; $4=$ minimal $/$ insufficient; $5=$ none. Voiding function was analyzed by comparing pre/post-TCAP AUASS. Statistical analysis utilized SAS software with $\mathrm{p}<0.05$ considered significant.

Results: After exclusions, 78 consecutive patients were analyzed with a mean age of 69.2 years and follow-up 39.8 months. Thirty-five (44.9\%) men reported pre-TCAP EF level of 1-2. Post-TCAP, 9 of $35(25.7 \%)$ regained EF of level 1-2 while $1(2.9 \%)$ achieved level 3 EF. Median pre-TCAP AUASS was 8.75 versus 7.50 postoperatively $(p=0.39)$. Six patients (7.7\%) experienced post-TCAP urinary incontinence. Lower pre-TCAP PSA $(\mathrm{p}=0.008)$ and higher Gleason sum $(\mathrm{p}=$ $0.002)$ were associated with higher post-TCAP AUASS while prostate volume demonstrated a trend $(p=0.07)$. Post-TCAP $\mathrm{EF}$ and stable AUASS were not associated with increased disease-recurrence $(\mathrm{p}=0.24$ and $\mathrm{p}=0.67$, respectively).

Conclusions: Stable voiding function was observed post-TCAP, with an overall incontinence rate of $7.7 \%$. Further, though erectile dysfunction is common following TCAP, $25.7 \%$ of previously potent patients demonstrated erections suitable for intercourse. While long-term data is requisite, consideration should be made for prospective evaluation of penile rehabilitation following primary TCAP.
\end{abstract}

Key words: prostatic neoplasms; cryoablation; erectile dysfunction; voiding dysfunction Int Braz J Urol. 2008; 34: 443-50

\section{INTRODUCTION}

With an expected 218,890 new cases and 27,050 deaths estimated in 2007, prostate cancer $(\mathrm{CaP})$ is the most common malignancy in men in the United States (1).With the ongoing stage migration of contemporary $\mathrm{CaP}$, patients diagnosed with apparent organ-confined tumors are faced with a spectrum of treatment modalities including surgical, radiation, medical and surveillance. All curative and palliative treatments for $\mathrm{CaP}$ are associated with some degree of morbidity, and the effects on quality of life (QOL) can be quite pronounced (2).

Targeted cryoablation of the prostate (TCAP) has emerged as an accepted therapy for both primary treatment of clinically localized $\mathrm{CaP}$, as well as for 
salvage therapy following failed definitive therapy, demonstrating survival and cancer-control at least equivocal to external-beam radiotherapy (3-7). Erectile dysfunction (ED) and voiding dysfunction are common following all potentially curative $\mathrm{CaP}$ treatments. ED is reported to be particularly pronounced following TCAP due to the hypothermic impact of the ice ball on the peri-prostatic nerves. However, since no ligation of the neurovascular bundle occurs during TCAP, the potential for axonal regeneration exists (8). As such, several series have reported varying degrees of erectile function (EF) recovery following TCAP (3,9-13). Additionally, "nerve-sparing" TCAP has shown encouraging results with regards to recovery of EF $(14,15)$.

In addition to effects on EF, conflicting reports of the impact TCAP has on voiding function and lower urinary tract symptoms (LUTS) have been documented $(3,5,9-12)$. We investigated our experience with primary TCAP in an attempt to determine $\mathrm{EF}$ and voiding function outcomes, as well as predictive factors for improved results, in patients treated for clinically localized $\mathrm{CaP}$.

\section{MATERIALS AND METHODS}

After obtaining institutional review board approval, we retrospectively reviewed all patients with biopsy-proven $\mathrm{CaP}$ treated with TCAP between 2/2000-5/2006 at our institution. Patients receiving salvage TCAP and those with incomplete pre- or postTCAP EF or voiding function data were excluded. All procedures were performed utilizing the Cryocare prostate cryoablation system (Endocare, Inc., Irvine, CA) with real-time transrectal-ultrasound monitoring and a brachytherapy grid for probe/thermosensor placement as has been previously described.(3, 10, 11) All procedures utilized a urethral warmer and employed a six-cryoprobe technique, implementing a double-freeze-thaw cycle.

Clinicopathologic variables included age at TCAP, race, body mass index (BMI), pre-TCAP prostate specific antigen (PSA), Gleason sum (GS), clinical stage, prostate volume, receipt of neoadjuvant androgen deprivation therapy (NADT), and pre-TCAP EF and American Urological Associa- tion Symptom Score (AUASS) assessment. NADT was typically employed to downsize glands $>40$ $\mathrm{cm}^{3}$. Pre and post-TCAP EF scores and AUASS were recorded at last pre-TCAP follow-up and compared to scores at last post-TCAP follow-up. AUASS were compared as continuous variables, as well as divided categorically according to AUA guidelines: mild (0-7, Category 1), moderate (8-19, Category 2) and severe (20-35, Category 3) (16). Urinary incontinence was defined as any degree of urine leakage, and the number of pads (if any) was recorded. Patients were followed-up with a history/ physical examination, PSA, EF score, and AUASS every 3 months for 2 years, every 6 months for the next 2 , and annually thereafter. EF was documented using a 5-point scale as follows: $1=$ fully potent, $2=$ erections sufficient for intercourse, $3=$ partial erections insufficient for intercourse, $4=$ minimal erections insufficient for intercourse and $5=$ no erections. Post-TCAP ED therapy was noted: including phosphodiesterase type-5 inhibitors (PDE5i), prostaglandin E1 analogues (PGE1), vacuum erection device (VED), and inflatable penile prosthesis (IPP). Notably, it was not our routine practice during the study period to encourage penile rehabilitation postTCAP. Disease-recurrence/progression was defined as: biochemical (BCR) according to the American Society for Therapeutic and Radiology and Oncology (ASTRO) criteria (17), biopsy-proven local recurrence (LCR), distant metastasis, by initiation of salvage ADT (SADT) for rising PSA, or by CaPrelated death.

Data analysis utilized Student's-t-test, Chi square and Kruskal-Wallis analysis of variance (where appropriate), as well as univariate/multivariate logistic regression, with all potential explanatory covariates incorporated into models. Independent variables were modeled as continuous and categorical variables as follows: age $\geq 70$ vs. $<70$ years, Gleason grade sum $\geq$ 7 vs. $<7$, PSA $\geq 10$ vs. $<10 \mathrm{ng} / \mathrm{mL}$, prostate volume $\geq 30$ vs. $<30 \mathrm{~cm}^{3}$ and $\mathrm{BMI} \geq 30$ vs. $<30 \mathrm{~kg} / \mathrm{m}^{2}$. All p-values were based on 2-sided tests of significance, with $p<0.05$ considered statistically significant. The Hosmer-Lemeshow test eliminated models that fit poorly. Statistical analysis utilized SAS computerized software, version 9.1 (SAS Institute Inc., Cary, $\mathrm{NC})$. 


\section{RESULTS}

Demographic data and disease characteristics are outlined in Table-1. After exclusions, 78 consecutive patients were analyzed with a mean age of 69.2 years (range: 55.3 - 80.9), pre-TCAP PSA of 9.4 $\mathrm{ng} / \mathrm{mL}$ (range: 0.8 - 84.0), Gleason grade sum of 6.5 (range: 3 - 9), and prostate volume of $29.7 \mathrm{~cm}^{3}$ (range: $10-50)$.

\section{Erectile Function}

Overall, 61 (78.2\%) patients reported some degree of pre-TCAP EF with or without the use of erectile aids. However, only 35 (of $61,57.4 \%$ ) reported pre-TCAP erections sufficient for intercourse (EF levels 1 or 2$)$. Seventeen $(21.8 \%)$ patients reported pre-TCAP impotence and 2 of these underwent postTCAP IPP. At a mean follow-up of 39.8 months (range: 0.6-92.4), 10 (16.4\%) patients regained EF; $9(14.8 \%)$ achieving level 2 EF with PDE5i only (n $=3)$, VED only $(n=2)$, or PDE5i/VED $(n=4)$ and 1 (1.6\%) achieving level 3 EF with PDE5i/VED. Subset analysis of the 35 men who were previously potent (EF level 1-2), however, demonstrated the post-TCAP EF recovery rate to be $25.7 \%$. The mean time to potency restoration was 15.2 months (range: 9.7-29.3). Notably, the 2 patients who underwent IPP were not regarded as potent post-TCAP. No significant clinical predictors of post-TCAP EF were identified on either univariate or multivariate analysis (data not shown). Post-TCAP EF was not associated with an increased risk of disease-recurrence $(p=0.24)$. EF outcomes are outlined in Table- 2 .

\section{Voiding Dysfunction}

Median pre-TCAP AUASS was 8.75 (range: $0-31.0$ ) vs. 7.50 (range: $0-33.0$ ) postoperatively and did not change with treatment $(\mathrm{p}=0.39)$. When analyzed categorically, $34(43.6 \%)$ men reported Category 1, $37(47.4 \%)$ reported Category 2, and $7(9.0 \%)$ reported Category 3 LUTS. Post-TCAP, 39 (50.0\%), $33(42.3 \%)$, and $6(7.7 \%)$ men reported categories 1 , 2 , and 3 LUTS, respectively. There was no difference between pre and post-TCAPAUASS when compared categorically $(p=0.74)$. Six patients $(7.7 \%)$ experi- enced urinary incontinence at last follow-up: 1 (1.3\%) requiring $0-1$ pads/day, $1(1.3 \%)$ requiring $1-2$ pads/ day, $2(2.6 \%)$ requiring $2-3$ pads/day and $1(1.3 \%)$ who developed a bladder neck contracture, which was dilated, resulting in $>3$ pads/day incontinence. Overall, 46 patients $(59.0 \%)$ demonstrated improved or stable AUASS, while 32 (41.0\%) reported worsening LUTS. Importantly, the presence of post-TCAP improved/stable AUASS was not associated with an increased risk of cancer recurrence $(p=0.67)$. Voiding function findings are outlined in Table-3.

On logistic regression (Table-4), lower preTCAP PSA (Odds Ratio (OR) 3.06; $\mathrm{p}=0.008$ ) and higher Gleason sum (OR 3.80; $p=0.002$ ) were associated with higher post-TCAP AUASS. Larger preTCAP prostate volume demonstrated a trend towards worsening LUTS and higher post-TCAP AUASS outcomes $(p=0.07)$. However, receipt of NADT did not demonstrate a relationship with post-TCAPAUASS $(\mathrm{p}=0.67)$. No patients underwent a pre or post-TCAP transurethral resection/ablation of the prostate for urinary retention or LUTS during the study period.

\section{Cancer-Control/Disease-Progression}

Thirteen (16.7\%) patients demonstrated disease-progression: 10 (76.9\%) BCR, 2 (15.4\%) LCR, and $1(7.7 \%)$ SADT. No CaP-related deaths were noted during the study period. Mean time-to-recurrence was 11.9 months (median 9.9; range: 5.7-23.8). On Kaplan-Meier analysis, BCR-free survival was $97.9 \%$ at 1 year, $95.7 \%$ at 3 years and $82.9 \%$ at 5 years. Progression-free survival was $97.9 \%$ at 1 year, $95.7 \%$ at 3 years, and $71.1 \%$ at 5 years. Four $(5.1 \%)$ patients died of unrelated causes and were censored at the time of death. Overall survival was $95.9 \%$ at 1 year, $94.3 \%$ at 3 years, and $94.3 \%$ at 5 years.

Multivariate categorical analysis demonstrated African American race (OR 4.46, $\mathrm{p}=0.03$ ), GS $\geq 7(\mathrm{OR} 6.4, \mathrm{p}=0.02)$, pre-TCAP PSA $\geq 10 \mathrm{ng} / \mathrm{mL}$ (OR 3.82, $\mathrm{p}=0.002)$, and age $\geq 70$ years (OR 2.74, $\mathrm{p}=0.01)$ to predict disease-recurrence, while NADT administration trended towards decreased recurrence (OR 2.23, $\mathrm{p}=0.05$; Table-5). On continuous variable analysis; however, only age (OR 2.74, $\mathrm{p}=0.01)$ remained a predictor of disease-progression (Table$6)$. 


\section{Outcomes Following Primary Targeted Prostate Cryoablation}

Table 1 - Demographic and clinicopathologic data on 78 men undergoing primary targeted cryoablation of the prostate (TCAP) for clinically localized prostate cancer.

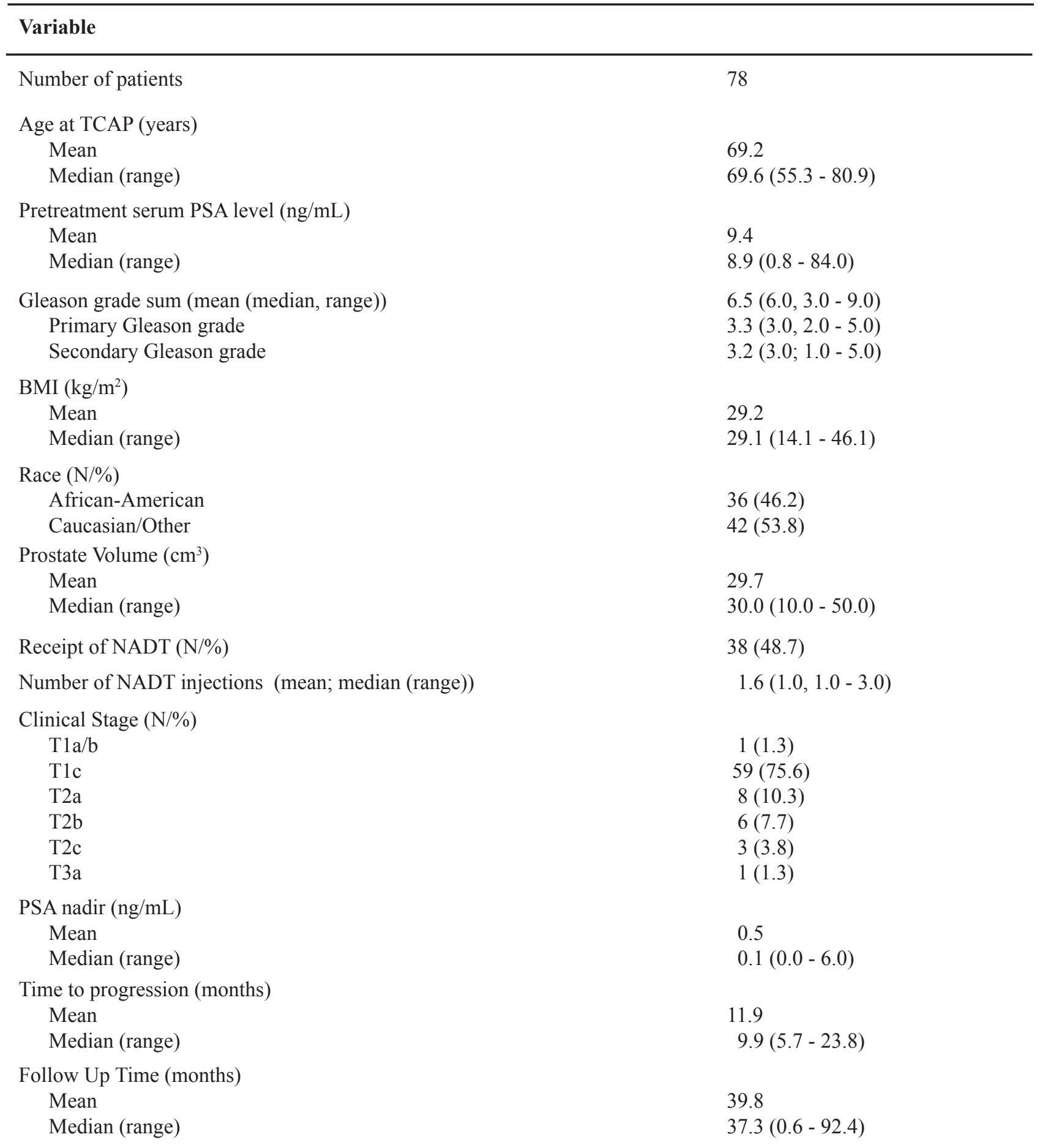

$N A D T=$ neoadjuvant androgen deprivation therapy. 


\section{Outcomes Following Primary Targeted Prostate Cryoablation}

Table 2 - Distribution of pre- and post- targeted cryoablation of the prostate (TCAP) erectile function and dysfunction.

\begin{tabular}{lcc}
\hline EF Level & Pre-TCAP $(\mathrm{N} / \%)$ & Post-TCAP $(\mathrm{N} / \%)$ \\
\hline 1 & $9(11.5)$ & $0(0)$ \\
2 & $26(33.4)$ & $9(11.5)$ \\
3 & $17(21.8)$ & $1(1.3)$ \\
4 & $9(11.5)$ & $0(0)$ \\
5 & $17(21.8)$ & $68(87.2)$ \\
\hline
\end{tabular}

\section{COMMENTS}

TCAP has steadily gained popularity for primary and salvage treatment of clinically localized $\mathrm{CaP}$ $(3,7)$. Erectile and voiding dysfunctions are common following all potentially curative $\mathrm{CaP}$ therapies. As these morbidities continue to be elucidated, there is increasing interest in improving QOL outcomes for men undergoing CaP-directed treatments, with particular emphasis on ED and voiding outcomes. However, there remains both a paucity of data focusing on these outcomes, as well as considerable variability following primary TCAP $(3,5-7,9,11,13)$. Particular to EF, the thought that TCAP resulted in irreversible ED secondary to hypothermic injury to the cavernous nerves is being questioned. The potential for axonal regeneration after TCAP-related neuropraxia lends credence to reports of EF recovery following TCAP (8).

Bahn et al. reported 7-year outcomes on these endpoints in 590 men undergoing primary TCAP. Of 373 men potent pre-TCAP, only 19 (5.1\%) recovered potency at an average of 16.4 months post-TCAP. Regarding voiding function, of 533 previously continent patients, 448 (84.1\%) regained continence at an average of 6.1 months (3). In another series, Han et al.

Table 3 -Analysis of pre- and post-targeted cryoablation of the prostate (TCAP) American Urologic Association Symptom Score (AUASS).

\begin{tabular}{cccc}
\hline Variable & Pre-TCAP & Post-TCAP & p Value \\
\hline AUASS & & & \\
Mean & 9.96 & 9.11 & 0.39 \\
Median (range) & $8.75(0.0-31.0)$ & $7.50(0.0-33.0)$ & \\
AUASS Category (N/\%) & & & 0.75 \\
Category 1 & $34(43.6)$ & $39(50.0)$ & \\
Category 2 & $37(47.4)$ & $33(42.3)$ & \\
Category 3 & $7(9.0)$ & $6(7.7)$ & \\
\hline
\end{tabular}

Table 4 - Logistic regression analysis for predicting worsening post- targeted cryoablation of the prostate (TCAP) lower urinary tract symptoms (i.e. increased American Urologic Association Symptom Score).

\begin{tabular}{lcc}
\hline Variable & Odds Ratio & p Value \\
\hline Age $($ years $)$ & 1.68 & 0.112 \\
Pre-TCAP PSA $(\mathrm{ng} / \mathrm{mL})$ & 3.05 & 0.008 \\
BMI $\left(\mathrm{kg} / \mathrm{m}^{2}\right)$ & 0.66 & 0.516 \\
Prostate volume $\left(\mathrm{cm}^{3}\right)$ & 1.93 & 0.070 \\
Gleason sum & 3.80 & 0.002 \\
\hline
\end{tabular}


Table 5 - Logistic regression analysis for predicting disease-recurrence utilizing categorical variable modeling.

\begin{tabular}{lcc}
\hline Variable & Odds Ratio & p Value \\
\hline Age $\geq 70$ years (vs. $<70)$ & 2.74 & 0.01 \\
African-American race (vs. other) & 4.46 & 0.03 \\
Pre-TCAP PSA $\geq 10 \mathrm{ng} / \mathrm{mL}$ (vs. $<10)$ & 3.82 & 0.002 \\
BMI $\geq 30 \mathrm{~kg} / \mathrm{m}^{2}($ vs. $<30)$ & 1.10 & 0.66 \\
Prostate volume $\geq 30 \mathrm{~cm}^{3}$ (vs. $\left.<30\right)$ & 0.84 & 0.95 \\
Gleason sum $\geq 7$ (vs. $<7)$ & 6.40 & 0.02 \\
Receipt of NADT (vs. none) & 2.23 & 0.05 \\
\hline
\end{tabular}

$T C A P=$ targeted cryoablation of the prostate; NADT = neoadjuvant androgen deprivation therapy.

Table 6 - Logistic regression analysis for predicting disease-recurrence utilizing continuous variable modeling.

\begin{tabular}{lcc}
\hline Variable & Odds Ratio & p Value \\
\hline Age (years) & 2.74 & 0.01 \\
Pre-TCAP PSA (ng/mL) & 0.90 & 0.37 \\
BMI (kg/m2) & 0.77 & 0.45 \\
Prostate volume $\left(\mathrm{cm}^{3}\right)$ & 0.70 & 0.49 \\
Gleason sum & 2.44 & 0.02 \\
\hline
\end{tabular}

reported on 106 patients undergoing primary TCAP. They observed impotence rates of $87 \%$ for previously potent men, while $3(3 \%)$ required pads for urinary incontinence (5). Similarly, Polascik et al. reported on 50 men undergoing primary TCAP, documenting a response rate of $50 \%$ ( 3 of 6 previously potent men) with the use of PDE5i therapy after TCAP. Further, they found a $3.7 \%$ rate of post-TCAP incontinence, requiring 1-2 pads/day (6). In a questionnaire-based study, Anastasiadis et al. reviewed 131 men undergoing primary or salvage TCAP. They found that the most bothersome symptoms following TCAP were sexual, followed by urinary complaints. In particular, ED (90\% vs. $86 \%)$ and incontinence rates $(10 \%$ vs. $5.9 \%$ ) were significantly worse in the salvage versus primary TCAP groups, respectively (9).

Our series demonstrated a recovery rate of EF suitable for intercourse of $25.7 \%$ in previously potent men ( $14.8 \%$ overall), with responses to VED, PDE5i, or both in combination (Table-2). It is important to reiterate that it was not our general practice to actively pursue or recommend penile rehabilitation in these patients. Therefore, we infer the potential for further improvement in EF outcomes with implementation of penile rehabilitation protocols post-TCAP. In fact, Ellis et al. reported a recent series of 416 consecutive men undergoing primary TCAP whereby daily VED use was recommended (without constriction ring) beginning 6 weeks post-TCAP for previously potent men along with PDE5i every other day and as needed beginning 6 months post-TCAP (11). They documented progressive EF recovery with this protocol: $29.1 \%$ regaining EF at 1 year, $48.5 \%$ at 2 years, and $51.3 \%$ at 4 years. Similarly, encouraging findings of EF recovery have been documented in other series (13), as well as our own (25.7\%), even in the absence of penile rehabilitation. These findings have prompted us to adopt a regimen of aggressive penile rehabilitation following TCAP.

Regarding voiding outcomes, we identified urinary incontinence rates comparable to most series, with $7.7 \%$ of men reporting some degree of urine leakage (regardless of pad usage) (3,5,9-12). However, pre-TCAP continence was not recorded consistently in our cohort. Thus, we considered all men to be fully continent prior to TCAP, which may be an overestimation in this regard. Since considerable variations in incontinence definitions exist in the literature, we employed a strict definition of post-TCAP incontinence as any degree of leakage (regardless of pad usage) in order to capture any patient with this complaint. 
Our series demonstrated oncologic outcomes similar to most contemporary literature $(3,5,6,10,11,13)$. Specific to our series, it is noteworthy that improved EF or voiding function demonstrated no association with increased risk for cancer recurrence ( $p=0.24$ and $p=0.67$, respectively). In other words, a suboptimal freeze cannot explain the outcomes seen in our cohort. Another unique finding of our analysis was the increased risk of disease-progression in African-American patients (compared to others) on multivariate analysis (OR 4.46, $\mathrm{p}=0.03$ ). While previously reported in radiation and prostatectomy series $(18,19)$, to our knowledge, ours is the first series documenting this association in men undergoing primary TCAP.

An additional novel feature of our series is the use of validated objective assessments of LUTS (AUASS) (16). To our knowledge, no prior series has utilized this instrument for LUTS comparisons in men undergoing TCAP. Our series demonstrated stable AUASS, whether analyzed as continuous or categorical variables. Regression analysis demonstrated worsening AUASS to be associated with lower pre-TCAP PSA and higher Gleason sum (Table-4). While the significance of this remains unclear, we hypothesize that higher grade cancers that produce less PSA due to glandular de-differentiating may respond differently to the cryobiology of TCAP, potentially contributing to these findings. Notably, larger prostate volumes demonstrated a trend towards worsening post-TCAP LUTS, though this was not statistically significant $(\mathrm{p}=0.07)$. However, NADT administration did not demonstrate a significant relationship with postTCAPAUASS $(p=0.67)$. We suspect that with larger series and longer follow-up, a relationship between improved LUTS may be realized based on the ability of NADT to reduce the overall prostate volume, potentially offering improved voiding outcomes in this patient population, though we were unable to demonstrate this in our current series.

There are several limitations to this study. Firstly, we report a retrospective review of our findings at a single center and as such, our findings are subject to the inherent biases of this type of analysis. Consequently, patients were not evaluated in a prospective fashion using validated $\mathrm{EF}$ instruments such as the Sexual Health Inventory for Men or In- ternational Index of Erectile Function questionnaires to document objective pre- and post-TCAP EF. Additionally, our cohort remains relatively small $(n=78)$ with a somewhat short duration of follow-up (39.8 months). Further, selection bias may have occurred as we studied only patients with complete pre- and post-TCAP EF and AUASS data. Thus, these potential biases may limit the ability to demonstrate all potential relationships between variables and the endpoints of the study.

Nonetheless, we feel this data is compelling enough to further investigate the possibility of improving EF outcomes following primary TCAP. Penile rehabilitation has proven useful following radical prostatectomy (20), and results appear encouraging following TCAP, though data is limited (11). For these reasons, we have integrated this strategy into our pre-operative and postTCAP treatment protocol, utilizing validated questionnaires to objectively determine our outcomes.

With regards to LUTS, TCAP does not seem to improve nor worsen symptoms to any significant degree based on our results. However, a discussion of the potential for urinary incontinence is paramount, as this remains a considerable bother to patients who experience this complaint following TCAP (9).

\section{CONCLUSIONS}

Primary TCAP resulted in stable postoperative AUASS, while ED remains common. However, $25.7 \%$ of previously potent men demonstrated EF suitable for successful intercourse in the absence of penile rehabilitation. Neither the restoration of EF, nor the presence of stable/improved LUTS were associated with disease-recurrence and therefore, not a result of suboptimal cryoablation. While long-term, prospective data employing validated instruments is requisite, implementation of a proactive penile rehabilitation protocol should be considered in order to maximize sexual outcomes following primary TCAP.

\section{ACKNOWLEDGEMENT}

Kimberly D. Lamar, Ph.D. provided statistical analysis of this study. 


\section{CONFLICT OF INTEREST}

None declared.

\section{REFERENCES}

1. Jemal A, Siegel R, Ward E, Murray T, Xu J, Thun MJ: Cancer statistics, 2007. CA Cancer J Clin. 2007; 57: 43-66.

2. Penson DF, Litwin MS: Quality of life after treatment for prostate cancer. Curr Urol Rep. 2003; 4: 185-95.

3. Bahn DK, Lee F, Badalament R, Kumar A, Greski J, Chernick M: Targeted cryoablation of the prostate: 7 -year outcomes in the primary treatment of prostate cancer. Urology. 2002; 60: 3-11.

4. Chin JL, Ng CK, Touma NJ, Pus NJ, Hardie R, Abdelhady M, et al.: Randomized trial comparing cryoablation and external beam radiotherapy for T2C-T3B prostate cancer. Prostate Cancer Prostatic Dis. 2008; 11: 40-5.

5. Han KR, Cohen JK, Miller RJ, Pantuck AJ, Freitas DG, Cuevas CA, et al.: Treatment of organ confined prostate cancer with third generation cryosurgery: preliminary multicenter experience. J Urol. 2003; 170: 1126-30.

6. Polascik TJ, Nosnik I, Mayes JM, Mouraviev V: Shortterm cancer control after primary cryosurgical ablation for clinically localized prostate cancer using third-generation cryotechnology. Urology. 2007; 70: 117-21.

7. Wake RW, Hollabaugh RS Jr, Bond KH: Cryosurgical ablation of the prostate for localized adenocarcinoma: a preliminary experience. J Urol. 1996; 155: 1663-6.

8. Donnelly BJ, Saliken JC, Ali-Ridha N, Rewcastle JC, White LJ: Histological findings in the prostate two years following cryosurgical ablation. Can J Urol. 2001; 8: 1237-9.

9. Anastasiadis AG, Sachdev R, Salomon L, Ghafar MA, Stisser BC, Shabsigh R, et al.: Comparison of health-related quality of life and prostate-associated symptoms after primary and salvage cryotherapy for prostate cancer. J Cancer Res Clin Oncol. 2003; 129: 676-82.

10. Ellis DS: Cryosurgery as primary treatment for localized prostate cancer: a community hospital experience. Urology. 2002; 60: 34-9.

11. Ellis DS, Manny TB Jr, Rewcastle JC: Cryoablation as primary treatment for localized prostate cancer followed by penile rehabilitation. Urology. 2007; 69: 306-10.

12. Long JP, Bahn D, Lee F, Shinohara K, Chinn DO, Macaluso JN Jr: Five-year retrospective, multi-institutional pooled analysis of cancer-related outcomes after cryosurgical ablation of the prostate. Urology. 2001; 57 : 518-23.

13. Robinson JW, Donnelly BJ, Saliken JC, Weber BA, Ernst S, Rewcastle JC: Quality of life and sexuality of men with prostate cancer 3 years after cryosurgery. Urology. 2002; 60: 12-8.

14. Bahn DK, Silverman P, Lee F Sr, Badalament R, Bahn ED, Rewcastle JC: Focal prostate cryoablation: initial results show cancer control and potency preservation. J Endourol. 2006; 20: 688-92.

15. Lambert EH, Bolte K, Masson P, Katz AE: Focal cryosurgery: encouraging health outcomes for unifocal prostate cancer. Urology. 2007; 69: 1117-20.

16. Barry MJ, Fowler FJ Jr, O’Leary MP, Bruskewitz RC, Holtgrewe HL, Mebust WK, et al.: The American Urological Association symptom index for benign prostatic hyperplasia. The Measurement Committee of the American Urological Association. J Urol. 1992; 148: 1549-57; discussion 1564.

17. Horwitz EM, Vicini FA, Ziaja EL, Dmuchowski CF, Stromberg JS, Martinez AA: The correlation between the ASTRO Consensus Panel definition of biochemical failure and clinical outcome for patients with prostate cancer treated with external beam irradiation. American Society of Therapeutic Radiology and Oncology. Int J Radiat Oncol Biol Phys. 1998; 41: 267-72.

18. Powell IJ, Dey J, Dudley A, Pontes JE, Cher ML, Sakr W, et al.: Disease-free survival difference between African Americans and whites after radical prostatectomy for local prostate cancer: a multivariable analysis. Urology. 2002; 59: 907-12.

19. Tewari A, Horninger W, Pelzer AE, Demers R, Crawford ED, Gamito EJ, et al.: Factors contributing to the racial differences in prostate cancer mortality. BJU Int. 2005; 96: 1247-52.

20. Mulhall JP, Morgentaler A: Penile rehabilitation should become the norm for radical prostatectomy patients. J Sex Med. 2007; 4: 538-43.

Accepted:

May 9, 2008 